\title{
Robotic and Microrobotic Tools for Dental Therapy
}

\author{
Chen Cheng $\mathbb{D}^{1},{ }^{1}$ Xue Yinan $\mathbb{D}^{2},{ }^{2}$ Xie Zongxin, ${ }^{3}$ Shi Lei, ${ }^{1}$ Xue Yanan $\mathbb{D}^{4},{ }^{4}$ and Yang Yanli $\mathbb{D}^{1}$ \\ ${ }^{1}$ Stomatology Hospital, School of Stomatology, Zhejiang University School of Medicine, Zhejiang Provincial \\ Clinical Research Center for Oral Diseases, Key Laboratory of Oral Biomedical Research of Zhejiang Province, \\ Cancer Center of Zhejiang University, Hangzhou 310006, China \\ ${ }^{2}$ Biological Science, College of Chemistry and Life Sciences, Zhejiang Normal University, Jinhua 321000, China \\ ${ }^{3}$ Stomatology Hospital, Zunyi University School of Medicine, Zunyi 563000, China \\ ${ }^{4}$ Department of Plastic Surgery, Sir Run Run Shaw Hospital, Zhejiang University of Medicine, Hangzhou 310016, China
}

Correspondence should be addressed to Chen Cheng; 7319067@zju.edu.cn and Yang Yanli; yangyl_1982@163.edu.cn

Received 9 November 2021; Revised 2 January 2022; Accepted 18 January 2022; Published 18 February 2022

Academic Editor: Xian Wang

Copyright $(92022$ Chen Cheng et al. This is an open access article distributed under the Creative Commons Attribution License, which permits unrestricted use, distribution, and reproduction in any medium, provided the original work is properly cited.

Robotic and microrobotic tools such as dental operating microscopes and dental endoscopes are being used extensively in dental therapy, which have a significant impact on dental therapy and education. Herein, this paper reviews the state of the art of robotic and microrobotic tools for dental therapy. This article starts with a brief introduction of current robotic and microrobotic tools for dental therapy and then displays their applications in various dental problems; strengths and weaknesses are also surveyed. Lastly, the conclusion and outlook are discussed, referring to the emerging dental clinic problems and demands. This review is expected to provide guidelines for the therapeutic application of robotic and microrobotic tools and to promote the development of robots in dentistry.

\section{Introduction}

From a surgery perspective, the main symbol of the 21 st century is the progression of robotic surgery. Robots have developed rapidly in the field of medicine. They are mainly used to assist doctors to complete minimally invasive surgery or reach positions that are difficult to reach by hands. Robotics is a disruptive technology that will change diagnostics and treatment protocols in dental medicine. A robot device acts as a computer interface between the operator and the instrument. In some cases, this ensures that some surgeons who struggle with "straight" videoscopic operations face fewer technical challenges than traditional methods. The use of robotic tools influences what we can do evidently. For a long time, dental therapy has been hampered by the tiny space available in the mouth, making it difficult to see the surgical field. Unfortunately, robots are not used as extensively in dentistry as in medicine [1]. Few promising robotic systems are not yet available to dentists. Robotic therapy is developing slowly in dental medicine. Recent research shows that only a few authors with a dental or medical background were involved in articles about robots [2]. This omission truly harms the development and promotion of dental robots. Under these circumstances, robotic engineers have difficulty understanding the needs of dentists. Robotic and microrobotic tools have started to help dentists as magnification devices in therapy, but they are still difficult to automate. There is an urgent need to guide engineers to finally transform these robotic and microrobotic tools into robots. The development of microscopes toward the end of the 16th century started the use of robotic tools [3]. Robotic and microrobotic tools, including dental operating microscopes and dental endoscopes, are now often used [4, 5]. Robotic and microrobotic tools bring a series of advantages: they improve the dentists' operating position and prolong the dentists' professional life; the built-in light source illuminates the affected area and provides a clear surgical field of vision; the adjustable multiple magnifies the affected area and makes the structure of teeth visible, which provides the possibility for the accuracy of the operations [6, 7]. Dental microscopes were first used in endodontics and then widely used in various oral subspecies [6-8]. It seems that robotic 
and microrobotic tools can meet the needs of dentists, which robots cannot do. Nowadays, Three-Dimensional Robotic Digital Microscope is playing an implant role in neurosurgery [9]. A new robotic microscope is also reported in 2020 [10]. Robotic endoscopes are also in service in the gastrointestinal system [11]. These advanced robotic tools are one step closer to full automation. However, these robotic tools are still relatively low-end in dental treatment.

The utilization and development of robotic and microrobotic tools in endodontics and other oral subspecies will be discussed in depth in this study. The review will also focus on the needs of the dentists and current issues with robotic and microrobotic tools. We want to guide the development of dental robots according to the applications of these robotic and microrobotic tools.

\section{Robotic and Microrobotic Tools}

Robotic and microrobotic tools working as robotic imaging systems for dental clinical treatment include dental operating microscopes and dental endoscopes, as shown in Figures 1(a) and 1(b). The robotic system of endoscopes is shown in Figure 1(c).

2.1. Dental Operating Microscopes. Dental operating microscopes (DOMs) are stereomicroscopes. The microscope's left and right light paths provide different object angles to achieve a magnified three-dimensional image. The zoom system magnifies the image through multiple groups of lenses according to the selected magnification. A dental operating microscope can provide 2-30 times magnification, which can be adjusted according to doctors' demands [8]. A microscope can usually be used as the eye of a robot. Robots usually appear to complete a minimally invasive surgery, so microscopy is an indispensable part of robots. Previous studies show that the use of DOM among endodontics has increased significantly since the early 1990s; a survey in 2008 showed the frequency of DOM use by endodontics to be $90 \%$ [12]. Thus, the DOM is an integral tool in today's endodontic practice, which helps to optimize the visualization of the tooth and its substructures $[6,13]$. The benefits of DOM in nonsurgical and surgical endodontic procedures have been reported. Advantages include facilitating access preparation [14]; locating canal orifices [15]; enhancing fine motor skills [16]; improving the ability to examine, clean, and shape the complex canal anatomy; removing and bypassing separated instruments; detecting fracture lines; assisting in obturation; and improving surgical techniques [17-19]. Despite its advantages, DOM is far from an ideal magnifying tool for dental procedures. Currently, dental endoscopes are regarded as an alternative to microscopy.

2.2. Dental Endoscopes. Endoscopes are standard medical devices composed of an intraoral camera, an endoscope probe, and a computer. At present, an endoscope is one of the common tools for the application of microrobots. The common structure of an endoscope is shown in Figure 2. Despite the availability of these diagnostic devices, however, the observation of collateral root canals and fractures near the deep root canal area, the root apex, remains difficult. Moreover, dental operating microscopes only allow assessing areas close to the root canal entrance, and tomography does not provide the resolution required to identify fine structures and cannot be used under endodontic treatment. In contrast, endoscopes can allow highresolution and almost noninvasive observation inside the root canals under treatment, and they are under active research for various applications [20]. Previous studies have shown that dental endoscopes are a popular device to remove things falling into the sinuses, trachea, esophagus, or other cavities [21]. Thus, dental endoscopy has also been reported for implant surgery [22]. Historical studies have shown that endoscopy can assist periodontal surgery [23], joint surgery [24], bone marrow cavity observation [25], wound repair [26, 27], osteotomy [28], salivary lithotripsy [29, 30], biopsy [31, 32], polypectomy [33], stereotactic radiotherapy [34], tumor removal [35], and even nerve visualization [36]. It also has certain significance for oral distance teaching and telediagnosis [37]. Endoscopes can realize the microtransportation of light-curing materials, compare the effect of apical surgery, explore root canals, observe dental pulp, and realize root canal visualization [38-40]. In 2018, an endoscopic system based on an intraoral camera and image processing was reported [41]. This system is the first step to facilitating the observation of fine structures by intraoral cameras.

\section{Robotic and Microrobotic Tools in Dental Treatment}

3.1. Robotic and Microrobotic Tools in Endodontics. Endodontic therapy includes treating the infected dentin, enamel, and pulp of a tooth to remove the infection and the pain it causes. Successful endodontic therapy relies on mechanical and chemical cleansing of the entire infected space and its complete obturation with an inert filling material [42-44]. The different morphologies of the tooth structure lead to the unpredictable shape of the root canal system. In traditional endodontic therapy, dentists find the root canal by freehand and mouth mirrors. Although there are various auxiliary instruments, the chance of missing root canals is still hard to avoid. For example, the second mesiobuccal canal (MB2) of maxillary molars has a high probability of existence, but it is not easy to locate with the naked eye [45]. To increase the success of endodontic therapy, Pro. Apotheker and Jako invented the dental microscope in 1978. The first dental microscope was born in 1981 and is named dentiscope [46]. After 1993, dental microscopes became popular and were widely used in endodontic therapy. Currently, microrobotic tools are widely used in endodontic therapy [47].

3.1.1. Identify Caries and Cracked Teeth. The enamel will show early decay, microleakage, and a lack of dentin and enamel structural integrity at extreme magnification levels. Caries decayed in teeth, whose sensitivity of diagnosis was 


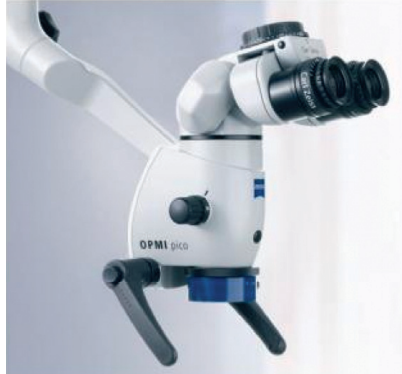

(a)

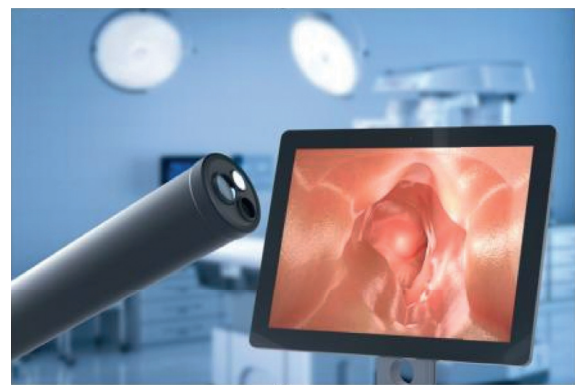

(b)

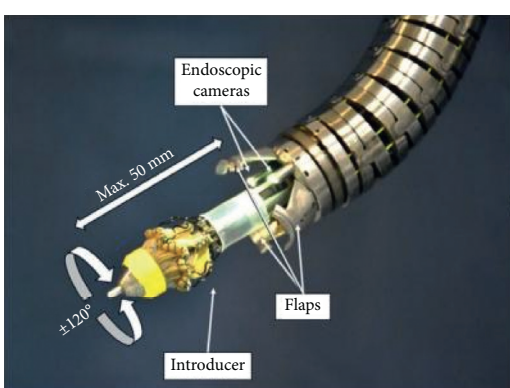

(c)

Figure 1: Robotic and microrobotic tools for dental treatments. (a) An operating microscope. (b) An endoscope. (c) A robotic manipulator of a robotic endoscopic system. Copyright 2019, Hindawi.

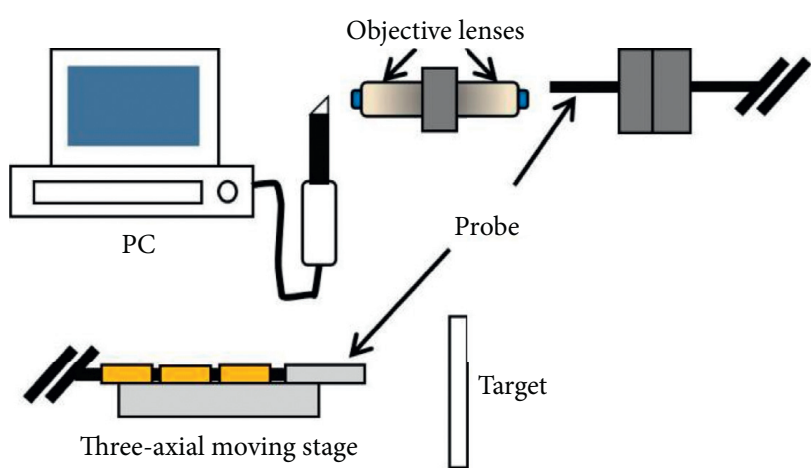

(a)

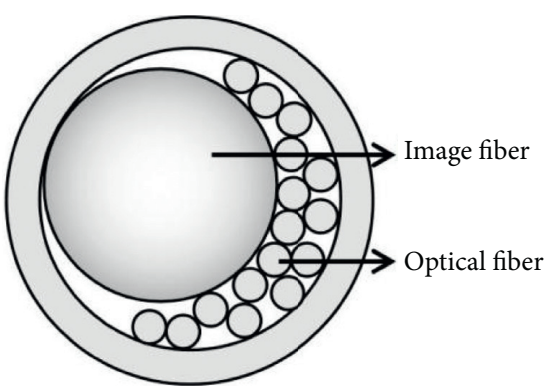

(b)

Figure 2: An endoscopic system. (a) A schematic diagram of an endoscopic system. (b) Cross-sectional schematic diagram of the probe.

significantly greater when magnification was used, as shown in Figure 3(a). Microrobotic tools also provide support for more precise fillings. In 2015, a miniature probe for delivery and monitoring of a photopolymerizable material was invented [48]. In addition, dentists diagnose cracked teeth by symptom-driven methods, as usual. The lack of visual confirmation always delays the diagnoses and therapies. Microleakage under a microscope will help dentists diagnose cracked teeth, as shown in Figures 3(b) and 3(c). An in vitro study showed that a fabricated root canal endoscope could facilitate detailed visualization of the apical foramen of a curved root canal and the fracture lines of the inside wall of a root canal [49].

\subsubsection{Root Canal Treatment}

(1) Location of Root Canals. Determining the location of root canals is the most critical and challenging step of root canal treatment. Missing canals more easily occur without microrobotic tools [50]. These missed or untreated canals were full of necrotic tissue and bacteria, leading to chronic symptoms and nonhealing periapical lesions. Root canal location is facing severe challenges because of the complexity of the root canal system. The ML canal in MB roots of maxillary molars can be tough to locate. ML canal detection was increased by adding microrobotic tools from $51 \%$ to
$82 \%$ in 39 test teeth [51]. The magnification of the operating field provided by the microscope and dental loupes is an essential factor in successfully locating the MB2canal, as shown in Figures 3(f) and 3(g) [52]. Figure 3(e) shows P2 of the anterior teeth, and Figure 3(h) shows ML2 of the posterior teeth. Keles et al. reported a comparative study on the accuracy of an endoscope to detect root canal anastomoses in mandibular molar teeth with microcomputed tomography. Divergence points located on the inner wall of main mesial root canals were undetectable under magnification via DOM, while endoscopic examination could detect $23.4 \%$ of them at the coronal halves of canals [53].

(2) Root Canal Preparation. The ideal endodontic treatment is based on adequate root canal preparation and suitable filling with inert filling materials. Root canal treatment should obtain proper root canal shape, with efficient cleaning performed before filling. However, factors such as root canal calcification and root canal variation and the anatomical structure of the complex root canal system often result in furcal perforation, ledge, canal transportation, strip perforation, root perforation, instrument separation, voids in the obturation, or underfilling or overfilling of the obturation, which leads to reinfection of the tooth. The application of microrobotic tools reduces teeth reinfection and promotes more accurate, minimally invasive root canal treatment [54]. It is not easy to distinguish root canal 
calcification from the naked eye. Microrobotic tools can magnify the difference in the color and texture between calcified and normal dentin, which provides powerful help for dredging small calcified root canals, as shown in Figures 3(i) and 3(j), taking the C-shaped root canal as an example of root canal variation. Because of the tortuous structure of the C-shaped root canal, pulp tissue or other debris will remain in the isthmus of the root canal during clinical root canal preparation, causing reinfection of the affected tooth and leading to the failure of root canal treatments [55]. In addition, C-shaped root canals are also prone to root canal perforation during preparation. As a serious complication, perforation, especially through the alveolar ridge, can cause abnormal communication between the pulp cavity and the periodontal tissue, which destroys the periodontal tissue, as shown in Figures 3(k) and 3(l). The microscope can not only illuminate the affected area but also allow the doctor to see the direction of the root canal more clearly based on the different colors of the root canal dentin and root canal perioral dentin, which is conducive to the good preparation of ultrasonic washing, chemical preparation, and other preparation techniques. Using microrobotic tools can not only reduce the occurrence of iatrogenic traumas such as furcal perforation, ledge, canal transportation, strip perforation, root perforation, instrument separation, voids in the obturation, or underfilling or overfilling of the obturation but can also be remedied when trauma occurs [56].

(3) Root Canal Filling. The analysis of the failure case indicated that the most common cause of failure was a leaky canal (30.4\%), followed by a missing canal (19.7\%), underfilling (14.2\%), anatomical complexity (8.7\%), overfilling (3.0\%), iatrogenic problems (2.8\%), apical calculus (1.8\%), and apical cracks (1.2\%) [57]. One of the primary causes of failed root canal treatment is an inadequate filling of the root canal system, in other words, the presence of gaps between the root canal filling material and the dentinal walls. Figures $3(\mathrm{~m})$ and $3(\mathrm{n})$ show incorrect root canal filling.

(4) Removal of Obstructions in the Root Canal. In root canal therapy, the original pulp is usually removed. If the instrument is separated and stuck in the middle of the root canal, doctors should remove the separated instrument from the root canal. Similar to the situation faced by root canal preparation, removing obstructions in the root canal becomes difficult due to the complex anatomy of root canal systems [58]. Clinically, microscopy is usually combined with ultrasound technology to prepare teeth for root canal retreatments. Under the microscope, the doctor can clearly distinguish the separation device, the plasticizing fluid, and the adhesive between the dowel and the root canal wall and then remove it by the ultrasound technique [59]. Figure 3(p) shows the separation device in the root canal.

3.1.3. Endodontic Microsurgery and Development of RobotAssisted Endodontic Therapy. Traditional periradicular surgery includes periradicular curettage, apicoectomy, and retrograde filling [60]. Microrobotic tools can make the operation more precise and more minimally invasive. In microsurgery, the microscope presents periapical lesions, which helps to accurately locate the root apex, collateral root canals, intercanal isthmus, missing root canals, and other structures, improves the surgical access and visibility of the apical area, and weakens the trauma caused by the operation at the same time. All of the above factors achieve the effect of precise preparation and tightly sealing the root apex, increasing the success rate of traditional apical surgery from $59.0 \%-71.9 \%$ to $91.7 \%-94.0 \%$ [61]. Iwai et al. showed in vitro training in endoscopic periradicular surgery using a printed three-dimensional model [62]. Garcia et al. reported a case of apical surgery of a maxillary molar by endoscopy [63]. A study compared microscopes versus endoscopes in root-end management. Researchers reported that microscopes and endoscopes were effective, and above $90 \%$ successful healing was achieved [20]. Figure 4 shows a case of the periradicular surgery conducted by the microscope and the endoscope. In addition to the use of robotic and microrobotic tools, robots and microrobots are also used in endodontic therapy. A robot was reported as a "vending machine" to provide the clinician with the required root canal therapy instruments during the procedure [64]. Janet Dong et al. reported an endodontic microrobot to complete root canal treatment automatically. However, this paper only described the preliminary development, including specifications and requirements, mechanical design, and controller systems [65]. In 2010, his further study described the design of a $Z$ axis actuator and tool quick change assembly in the micromachine for root canal treatment. However, further modification of the design may be needed after testing, and there are no longer any reports [66]. Microrobotic tools are now contributing to minimally invasive treatment rather than robotic treatment. Microrobots are the future direction of robot-assisted endodontic therapy. A recently published report suggested that a microrobot with catalytic ability could destroy the oral biofilm in the root canal and analyze the robot system in the laboratory. Figure 5 shows the CARs to act as a microrobot. In addition, the researchers explained the application of these robot systems in other applications, including the prevention of peri-implant infection or dental caries [67]. The use of robots in endodontics is very few. However, endodontic therapy is the most difficult to operate and needs the most robotic and microrobotic tools. Therefore, most of the context here is spent elaborating on the needs of endodontic doctors.

\subsection{Robotic and Microrobotic Tools in Periodontology. We} can define periodontal disease as an infectious bacterial disease resulting in an attack of tooth-supporting structures: bone, gums, cement, and the ligament system that anchors the tooth to the bone, including epithelial attachment [68]. This disease presents various degrees of severity, rates of progression, and responses to treatments. The most common means to diagnose periodontal disease is visual examination, assisted by a periodontal probe and radiographs. The periodontal probe gives a quantified reading of 

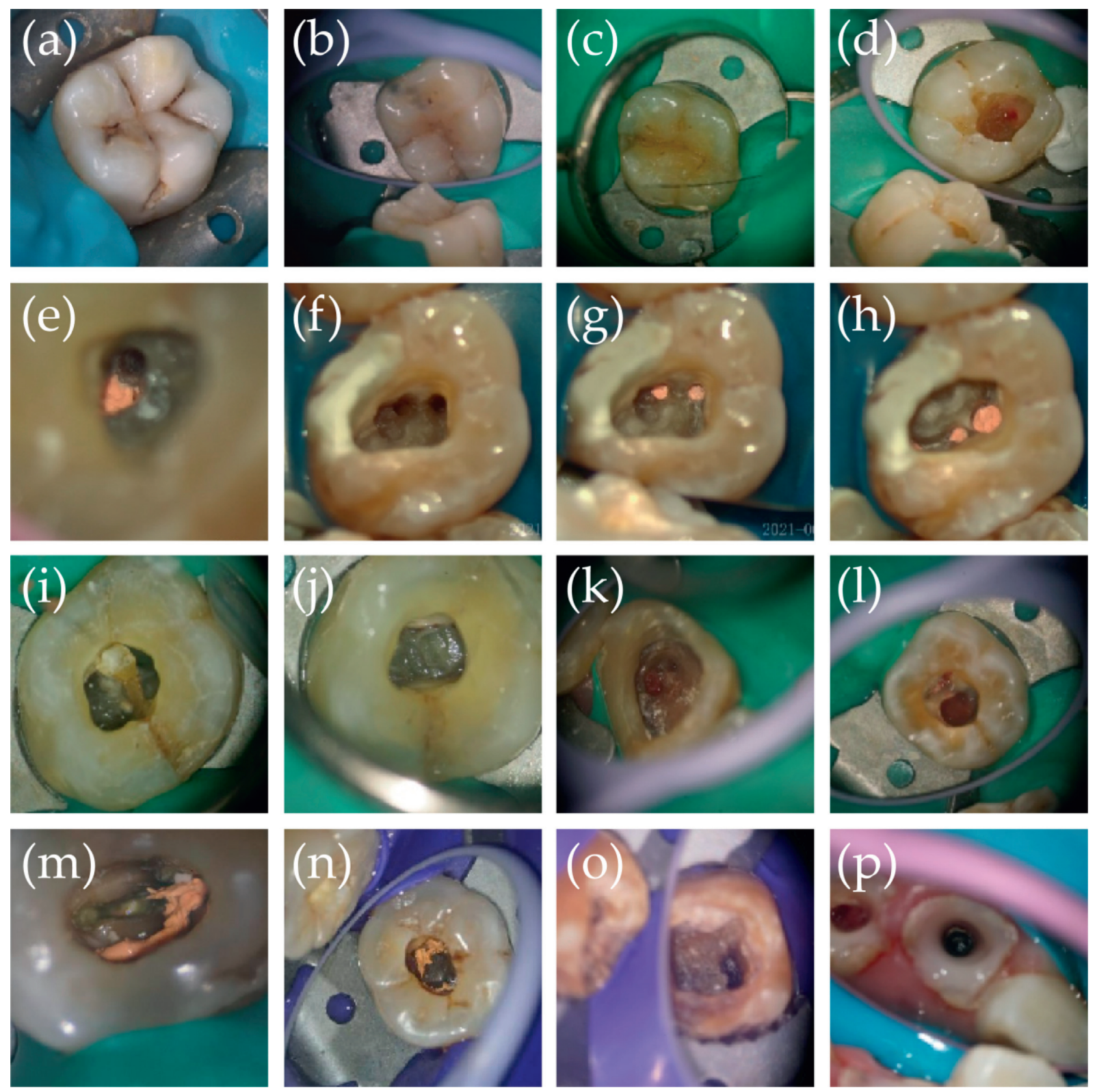

Figure 3: Photos in the endodontics therapy. (a) A decayed tooth. (b, c) A cracked tooth. (d) Deep caries infected pulp. (e) A tooth with P2. (f, g) A tooth with MB2. (h) A tooth with ML2. (i, j) Calcification of pulp cavity. (k, l) Destruction of pulp chamber bottom. (m, n) Root filling is incorrect. $(\mathrm{o}, \mathrm{p})$ Broken needle in root canal.

periodontal tissue damage, and the radiographs allow visualization of some of the structures not visible by direct vision, especially the interproximal bone [69]. In addition to subgingival plaque, subgingival calculus will also affect periodontal calculus, as a foreign body in the mouth will continue to stimulate and compress the periodontal calculus and promote the production or intensification of local periodontal tissue inflammation. Therefore, the removal of subgingival plaque and calculus has become the basis of periodontal treatment. Since the removal target is under the gums, the accuracy of periodontal surgery is required. Shanelec et al. found that dental calculus and inflammation coverage was significantly reduced after microscopy was used [70]. In summary, it is fully proven that microscope use, or magnification of the field of view, has positive effects on periodontal treatment.

In periodontal surgery, whether using sound waves, ultrasound, or manual techniques, it will inevitably have a destructive effect on the tooth's structure, causing deviations in operation. The application of magnifying equipment such as microscopes in periodontal treatment reduces this deviation, improves the accuracy of periodontal surgery, reduces periodontal surgery wounds, shortens the wound healing time, and reduces the formation of postoperative scars. Robotic and microrobotic tools have a significant effect on the transplantation of periodontal soft tissue flaps [71]. Dental endoscopes have already been used in periodontology and may provide 


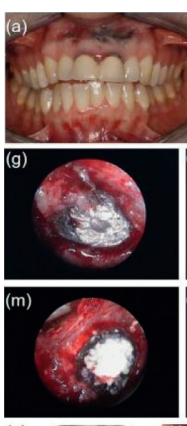

(b)
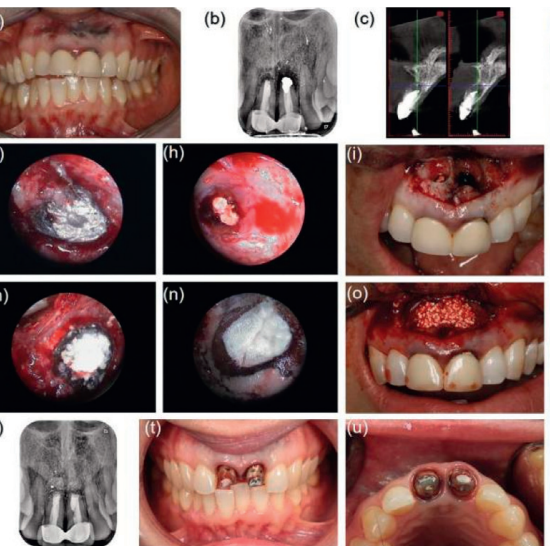
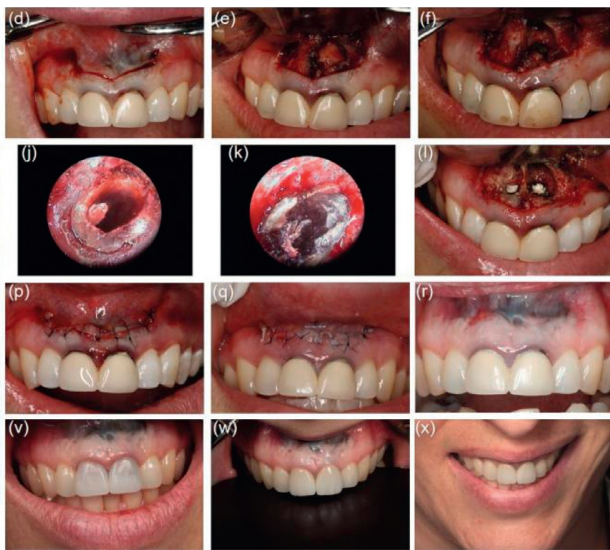

Figure 4: The periapical microsurgery with an endoscope and microscope.of two upper central incisors. (a) Preoperative clinical view. (b) X-ray showing low-density images around the end of roots. (c) CBCT showing the destruction of the buccal cortical layer of the left upper incisor. (d) Incision. (e) Raising of the full-thickness mucoperiosteal flap (f) Ostectomy and curettage of the periapical lesions. (g) Endoscopic view of the right upper incisor. (h) Endoscopic view of the left upper incisor. (i) Clinical view of the removal of the old filler material. (j) Endoscopic view of the right upper incisor after removal of the old filler material. (k) Endoscopic view of the left upper incisor after removal of the old filler material. (l) Clinical view of filling with MTA. (m) Endoscopic view of the right upper incisor after filling with MTA. (n) Endoscopic view of the left upper incisor after filling with MTA. (o) Bone transplantation with bone graft. (p) Suture the operation area. (q) 7 days after the operation. (r) 1 year after the operation. (s) X-ray showing good bone healing after 1 year. (t) After tooth preparation with the BOPT approach. (u) Occlusal view. (v) After installation of the provisional crowns. (w) After cementing the definitive crowns. (x) The smiling photo. Copyright 2020, Hindawi.

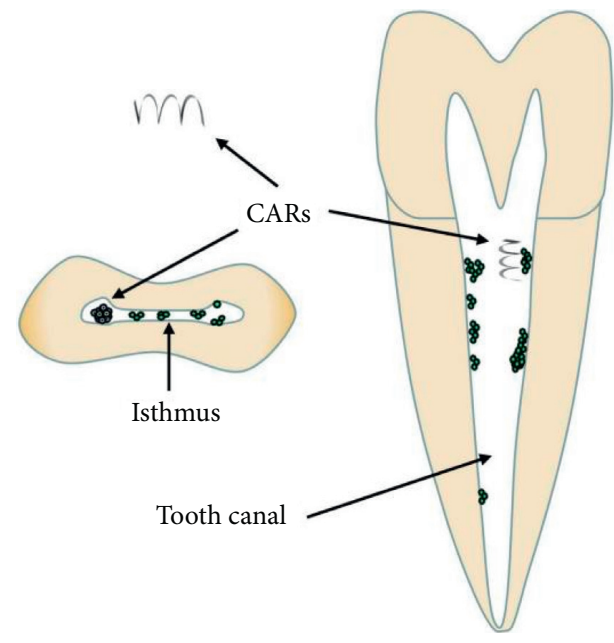

Figure 5: A magnetic microrobot for catalytic biofilm degradation.

additional benefits for calculus removal compared with traditional SRP [72].

Few robots are used in periodontology. Robots are only used to help clean teeth. Similarly, Ernst et al. developed a robot system to simulate the change in 3D brushing motion over time [73]. In vitro, experimental results show that the robot system can show repeatable significant differences in the cleaning effect of electric toothbrushes.

\subsection{Robotic and Microrobotic Tools in Oral and Maxillofacial} Surgery. Oral and maxillofacial surgery involves treatment of oral organs (teeth, alveolar bone, lips, cheeks, tongue, palate, pharynx, etc.), facial soft tissues, maxillofacial bones (upper jaw, mandible, cheekbone, etc.), surgical treatment of the temporomandibular joint, salivary glands, etc. [74-78]. The pathological section of surgical tumor resection was where the microscope was first used. Initially, microscopy was mainly used for postoperative observation of tumors or cysts. After the development of microsurgery technology, robotic and microrobotic tools have been widely used in oral surgery clinics: oral microsuturing can be used to suture small tissues such as blood vessels finely; oral microdiagnosis can identify cancerous tissues and cyst walls; oral microscopic resection of tumors can accurately locate the resection site and reduce trauma; oral microscopic pathological examination of the surgical margins ensures clean resection [79]. With the magnification of the field, robotic and microrobotic tools help doctors locate the lesion accurately and significantly reduce the wound area [80].

The application of microsurgery technology improves the success rate of free tissue transplantation into the recipient area. Surgeons can effectively repair maxillofacial injuries based on free transplantation, according to different vascularized body tissues. Oral and maxillofacial repair and reconstruction involve free tissue transplantation that requires anastomosis of blood vessels. Treatment should consider not only the prospect of tissue recovery but also aesthetics. According to research, the success rate of maxillofacial repair under microsurgery technology has significantly improved, and the incidence of complications has been reduced [81, 82]. Dentists can also use robotic and microrobotic tools for nerve repair, which is challenging to operate without magnification [83]. Endoscopic surgery of the temporomandibular joint is becoming an important research direction in treating the temporomandibular joint $[84,85]$.

In summary, the application and development of dental surgery microscopes promote the minimization of 
iatrogenic damage in surgical treatment, the minimization of medical costs, and the maximization of patient rehabilitation. Robots are also used in oral and maxillofacial surgery. In 2009, the US Food and Drug Administration approved the Da Vinci system for oral treatment of some malignant diseases [86] and all nonmalignant lesions of the oropharynx, even at the bottom of the throat and tongue. Robotassisted surgery can also provide good local control in the treatment of low-risk oral squamous cell carcinoma [87]. The oral application of this part is similar to the application of robots in surgery.

\subsection{Robotic and Microrobotic Tools in Oral Medicine.} There are many kinds of oral mucosal diseases with various causes. The diagnosis of mucosal diseases usually requires the observation of robotic and microrobotic tools. Doctors often need to take a biopsy or smear observation to diagnose mucosal disease. The mucosal disease can develop into cancer. After pathological examination, the cells need to be observed and confirmed by robotic and microrobotic tools [88-90]. The most important thing of mucosal diseases is the diagnosis, and the most common treatment is drug treatment. Therefore, microscopes and endoscopes can help diagnosis, but few robots appear to help treatment.

3.5. Robotic and Microrobotic Tools in Prosthodontics. The highest goal of prosthodontics is to make false teeth that mix spurious with genuine teeth. The most common products of prosthodontics are crowns, veneers, and inlays [91, 92]. If you want a natural last appearance of dentures, you need high polishing, good preparation. The finish line is the place where the prosthesis and the natural tooth are connected. If preparing the finish line is not fine enough, it will cause a series of problems and damage the stable environment in the oral cavity. The rough finish line means an insufficient connection between the prosthesis and the natural teeth, even a gap. Food residue and bacteria will accumulate in the gap, causing secondary caries. Imperfect shoulders can also lead to a protruding foreign body at the edge of the crown, which can stimulate the gums and cause complications such as gingival inflammation and bleeding [93]. Therefore, a continuous, clear, precise, smooth finish line will help improve the accuracy of the impression, significantly improving the adhesion between the final restoration and the preparation. It will also help to avoid stimulating periodontal soft tissue and prevent complications such as secondary caries caused by edge microleakage and loss of periodontal attachment and create a long-term stable oral environment. To monitor the effect of finish line preparation in real time, in addition to visual observation, a dental operating microscope can be used clinically. However, in recent years, after the concept of precise preparation was put forward, an increasing number of dentists began to pursue higher-precision preparation methods. Microscope operations gradually emerged in dental restorations: preliminary tooth preparation at low magnification; the rough parts are enlarged and finely polished one by one; polishing is carried out at high magnification to remove the fine cracks on the tooth preparation, forming a smooth tooth preparation. Dentists evaluated the accuracy of the impression with a microscope.

Most of the articles on prosthodontic robots are not exceeding the level of proof of concept, including the tootharrangement robots [94]. A tooth preparation robot system was invented and showed its clinical potential [95]. The report shows good results, but its results have not been verified in clinical settings thus far.

3.6. Robotic and Microrobotic Tools in Implant Dentistry. Robotic and microrobotic tools are widely used in implant dentistry. Limited by the patients' maxillary sinus bone quality, maxillary sinus lifting will be used in implant surgery to increase the success rate of implant surgery. Nevertheless, maxillary sinus lifting is not performed under direct vision, which may easily cause perforation of the sinus mucosa, leading to maxillary sinusitis. Endoscopic-assisted maxillary sinus lifting is a maxillary sinus lifting technique under endoscopic monitoring [22]. The doctor can use the endoscope inserted into the sinus through the canine fossa on the nasal side of the maxillary sinus to monitor the integrity of the maxillary sinus mucosa in real time during maxillary sinus lifting to better control the lifting height and the position of the graft material and provide timely feedback and rescue when perforation occurs, which dramatically reduces iatrogenic trauma and perforation probability.

For implant dentistry, robotic surgery is already a reality. In 2002, Boesecke et al. reported the first study of robotassisted dental implantation to minimize error [96]. In 2013, controlled and accurate drilling was achieved by a new robotic system [97]. In 2015, a 3-DOF robot which can detect and modulate the handpiece to ensure the accuracy of implantation was released [98]. The dental implant robots are not exceeding the level of proof of concept until 2017. In 2017, the Neocis Yomi robotic device (USA) became the world's first FDA-approved computer navigation robot system [99]. In 2017, The Autonomous Dental Implant Robot System was invented by the Fourth Military Medical University Hospital and Peking University [86]. The Autonomous Dental Implant Robotic System was approved by the National Medical Products Administration (NMPA) and clinical trials were under way. The robot system aims to prevent surgical errors and solve the problem of the lack of high-quality dentists in China.

\subsection{Robotic and Microrobotic Tools in Dental Photomicrog-} raphy and Oral Radiology. The initial application of dental photography in the clinic is mainly to record the anatomical shape and color characteristics of the soft and hard tissues of the oral cavity and maxillofacial region to provide support for doctors' clinical diagnosis, case records, formulation of treatment plans, and prognosis, effectively facilitating communication with doctors, patients, and colleagues. With the development of technology and the progress of the times, dental clinical operation is gradually refined and modernized. The demand for recording accurate dental operations has gradually increased, and dental photomicrography has 
TABLE 1: Strengths and weaknesses of robotic and microrobotic tools.

\begin{tabular}{lc}
\hline & Robotic and microrobotic tools \\
\hline Strengths & Weaknesses \\
Always a lighting system & Increase the cost \\
Appropriate magnification and more minimally invasive & Professional maintenance and regular maintenance \\
Reasonable ergonomic design & Lack the operating devices \\
More secure and efficiency & Special clinical operation training \\
Data record-keeping and teaching & \\
The realization of virtual reality & \\
\hline
\end{tabular}

also emerged. Compared with traditional photography, dental photomicrography is more suitable for shooting small parts such as root canals. It records the tiny parts of teeth more precisely and takes efficient, serial, and high-definition photos of the diagnosis and treatment process without affecting the treatment process [100].

It is generally believed that oral radiologists can penetrate almost all aspects of oral teeth in a minimally invasive manner. Burdea et al. designed a robot system for tooth subtraction photography, using a 6-DOF position sensor, and proposed a robot arm with an X-ray source [101].

\section{Strengths and Weaknesses}

The strengths and weaknesses of robotic and microrobotic tools are summarized in Table 1.

\subsection{Strengths}

(1) Most robotic and microrobotic tools have a lighting system.

(2) The naked eye resolution is only $0.2 \mathrm{~mm}$, while the dental operating microscope can be magnified 2-30 times. The limit resolution of the dental operating microscope can reach $0.006 \mathrm{~mm}$, significantly improving human eye resolution and obtaining more visual information to help with minimally invasive treatments [102]. In clinical operation, when adjusting magnification, there is no need to change the location of the microscope, and the stability of the field is good.

(3) Ergonomics is essential to the health of dentists. The investigation shows that more than $70 \%$ of clinicians whose long-term head anteversion angle is greater than 20 degrees will suffer from shoulder and neck pain. More than $85 \%$ of the clinicians who did not wear loupes had head anteversion greater than 30 degrees [103]. In addition, the use of a dental operating microscope can also achieve improved ergonomics at other levels. Using a dental operating microscope, the eyepiece and observation object distance are relatively stable, reducing unnecessary adjustment [104].

(4) Doctors and patients can maintain a relatively safe distance, reducing the risk of saliva and blood infection. The dental operating microscope can be equipped with an assistant mirror to facilitate the cooperation of the assistant during the operation of the microsurgery [105]. Skilled dentists and assistants can achieve more efficiency.

(5) Robotic and microrobotic tools can be equipped with a camera or a video camera and other image acquisition devices to realize real-time recording of the treatment process. Image data are conducive to doctor-patient communication so that patients can participate in developing treatment plans. It is also conducive to dental clinical teaching demonstration.

(6) The realization of virtual reality is expected to realize remote diagnosis and treatment [37].

\subsection{Weaknesses}

(1) The cost of robotic and microrobotic tools is high

(2) Robotic and microrobotic tools are precision instruments that need professional maintenance and regular maintenance

(3) Because of its use and operation complexity, operators and their assistants need special clinical operation training before use and need a certain adaptation period

(4) Robotic and microrobotic tools lack the operating devices that eventually become robots and microrobots

\section{Conclusion and Outlook}

Dentistry is moving toward a new world of robot-assisted and data-driven medicine. Judging from the dependence of stomatology on tools, stomatologists need practical tools very much. With the increasing use of robots and microrobot tools in fields other than endodontics, their importance in dental clinics has been gradually recognized. They amplify nuances that the human eye cannot see and the prospect of precise medical treatment. It is crucial for complex root canal exploration, cancer tissue excision, and other oral cavity procedures. In specific ways, they broaden the scope of oral clinics, promote the future of oral clinics, and strive to balance iatrogenic injuries and the rehabilitation of the afflicted region. Robotic and microrobotic tools provide several advantages in dental clinics. Nevertheless, the main drawbacks are the lack of cost-effectiveness produced by the high cost of microscopes and the technical sensitivity created by the difficulty of operating the microscope. We expect that the widespread use of microrobotic instruments will enable the implementation of an oral 
treatment regimen. Virtual reality robotic and microrobotic tool skill training will be accomplished soon. The reality is that robots are not widely used in dentistry. Robots may need to learn from the functions of these tools. What limits the development of dental robots? First, the development and use of robots are expensive. Second, robots systems are complex systems. At present, dental robots only do some simple work, but these microscopic tools can help doctors complete complex work, which may be one reason. Dentists' acceptance of robots depends largely on demand. On the other hand, the acceptance of robots to patients has not been improved, and patients are not ready for robotic surgery. In addition, the use of robots must rely on the critical input of data. However, with the development of the times, these problems will eventually be overcome. Oral treatment programs also include artificial intelligence, lowering the technical threshold for application and attaining clinical popularization. It is believed that in the near future, robotic microscopes and robotic endoscopes will spring up and occupy the market. This will also open the prelude for the robot to finally complete the treatment independently.

\section{Data Availability}

No data were used to support this study.

\section{Disclosure}

Chen Cheng and Xue Yinan are considered as the co-first authors.

\section{Conflicts of Interest}

The authors declare that they have no conflicts of interest.

\section{Authors' Contributions}

Chen Cheng and Xue Yinan contributed equally to this work.

\section{References}

[1] P. Ahmad and M. K. Alam, "Dental Robotics," A Disruptive Technology, vol. 21, 2021.

[2] T. C. T. van Riet, K. T. H. Chin Jen Sem, J. T. F. Ho, R. Spijker, J. Kober, and J. de Lange, "Robot technology in dentistry, part one of a systematic review: literature characteristics," Dental Materials: Official Publication of the Academy of Dental Materials, vol. 37, pp. 1217-1226, 2021.

[3] E. Allen and J. L. Turk, "Microscopes in the hunterian museum," Annals of the Royal College of Surgeons of England, vol. 64, pp. 414-418, 1982.

[4] G. Bocchialini, S. Negrini, A. Bolzoni Villaret, and L. Pianta, "Intraconal orbital displacement of a dental implant treated with an endoscopically-assisted approach," British Journal of Oral and Maxillofacial Surgery, vol. 58, pp. 469-471, 2020.

[5] B. Al Shaikhly, S. K. Harrel, M. Umorin, R. A. Augsburger, and P. Jalali, "Comparison of a dental operating microscope and high-resolution videoscope for endodontic procedures," Journal of Endodontics, vol. 46, pp. 688-693, 2020.

[6] M. Del Fabbro, S. Taschieri, G. Lodi, G. Banfi, and R. L. Weinstein, "Magnification devices for endodontic therapy," Cochrane Database of Systematic Reviews, vol. 8, Article ID Cd005969, 2015.

[7] M. Del Fabbro and S. Taschieri, "Endodontic therapy using magnification devices: a systematic review," Journal of Dentistry, vol. 38, pp. 269-275, 2010.

[8] H. S. Selden, "The dental-operating microscope and its slow acceptance," Journal of Endodontics, vol. 28, pp. 206-207, 2002.

[9] S. Maurer, V. Prinz, L. E. Qasem et al., "Evaluation of a novel three-dimensional robotic digital microscope (aeos) in neurosurgery," Cancers, vol. 13, 2021.

[10] J. T. Collins, J. Knapper, J. Stirling et al., "Robotic microscopy for everyone: the OpenFlexure microscope," Biomedical Optics Express, vol. 11, pp. 2447-2460, 2020.

[11] T. A. C. Visconti, J. P. Otoch, and E. L. A. Artifon, "Robotic endoscopy. A review of the literature," Acta Cirurgica Brasileira, vol. 35, Article ID e202000206, 2020.

[12] D. D. Kersten, P. Mines, and M. Sweet, "Use of the microscope in endodontics: results of a questionnaire," Journal of Endodontics, vol. 34, pp. 804-807, 2008.

[13] D. J. Bowers, G. N. Glickman, E. S. Solomon, and J. He, "Magnification's effect on endodontic fine motor skills," Journal of Endodontics, vol. 36, pp. 1135-1138, 2010.

[14] J. S. Mamoun, "The maxillary molar endodontic access opening: a microscope-based approach," European Journal of Dermatology, vol. 10, pp. 439-446, 2016.

[15] N. Pattanshetti, M. Gaidhane, and A. M. Al Kandari, "Root and canal morphology of the mesiobuccal and distal roots of permanent first molars in a Kuwait population--a clinical study," International Endodontic Journal, vol. 41, pp. 755-762, 2008.

[16] B. G. Khayat, "The use of magnification in endodontic therapy: the operating microscope," Practical periodontics and aesthetic dentistry: Practical Procedures and Aesthetic Dentistry, vol. 10, pp. 137-144, 1998.

[17] S. I. Kratchman, "Endodontic microsurgery," Compendium of continuing education in dentistry, vol. 28, pp. 399-405, 2007.

[18] P. Mines, R. J. Loushine, L. A. West, F. R. Liewehr, and J. R. Zadinsky, "Use of the microscope in endodontics: a report based on a questionnaire," Journal of Endodontics, vol. 25, pp. 755-758, 1999.

[19] S. Kim and S. Kratchman, "Modern endodontic surgery concepts and practice: a review," Journal of Endodontics, vol. 32, pp. 601-623, 2006.

[20] S. Taschieri, M. Del Fabbro, T. Testori, and R. Weinstein, "Microscope versus endoscope in root-end management: a randomized controlled study," International Journal of Oral and Maxillofacial Surgery, vol. 37, pp. 1022-1026, 2008.

[21] M. Ouzhu, C. Wu, L. Ye, J. Dawa, and B. Hu, "Endoscopic removal of dental prosthesis impacted in the duodenal papilla," Endoscopy, vol. 51, pp. E10-e11, 2019.

[22] O. Nahlieli, J. Moshonov, A. Zagury, E. Michaeli, and N. Casap, "Endoscopic approach to dental implantology," Journal of Oral and Maxillofacial Surgery: Official Journal of the American Association of Oral and Maxillofacial Surgeons, vol. 69, pp. 186-191, 2011.

[23] T. G. Wilson, S. K. Harrel, M. E. Nunn, B. Francis, and $\mathrm{K}$. Webb, "The relationship between the presence of toothborne subgingival deposits and inflammation found with a dental endoscope," Journal of Periodontology, vol. 79, pp. 2029-2035, 2008.

[24] M. J. Troulis, W. B. Williams, and L. B. Kaban, "Endoscopic mandibular condylectomy and reconstruction: early clinical results," Journal of Oral and Maxillofacial Surgery: Official Journal of the American Association of Oral and Maxillofacial Surgeons, vol. 62, pp. 460-465, 2004. 
[25] W. Engelke, M. Lazzarini, W. Stühmer, and V. Beltrán, "Support immersion endoscopy in post-extraction alveolar bone chambers: a new window for microscopic bone imaging in vivo," PLoS One, vol. 10, Article ID e0145767, 2015.

[26] A. Sinha and S. Natarajan, "Comparative evaluation of clinical and radiological outcomes of retromandibular transparotid and transoral endoscopic-assisted approach for surgical management of mandibular subcondylar fractures," Craniomaxillofacial Trauma \& Reconstruction, vol. 14, pp. 90-99, 2021.

[27] A. M. Strohl and R. M. Kellman, "Current management of subcondylar fractures of the mandible, including endoscopic repair," Facial plastic surgery clinics of North America, vol. 25, pp. 577-580, 2017.

[28] M. E. Papadaki, L. B. Kaban, and M. J. Troulis, "Endoscopic vertical ramus osteotomy: a long-term prospective study," International Journal of Oral and Maxillofacial Surgery, vol. 43, pp. 305-310, 2014.

[29] P. Capaccio, S. Torretta, L. Pignataro, and M. Koch, "Salivary lithotripsy in the era of sialendoscopy," Acta Otorhinolaryngologica Italica: organo ufficiale della Societa italiana di otorinolaringologia e chirurgia cervico-facciale, vol. 37, pp. 113-121, 2017.

[30] G. Cammaroto, C. Vicini, F. Montevecchi et al., "Submandibular gland excision: from external surgery to robotic intraoral and extraoral approaches," vol. 26, pp. 853-857, 2020.

[31] E. J. Neiburger, "Endoscopic holography: a minimally invasive oral biopsy technique," Journal of the Massachusetts Dental Society, vol. 54, pp. 42-44, 2006.

[32] T. Nishizawa and N. Yahagi, "Endoscopic mucosal resection and endoscopic submucosal dissection: technique and new directions," Current Opinion in Gastroenterology, vol. 33, pp. 315-319, 2017.

[33] F. S. Salameh, "A promising new device for use in polypectomy," Endoscopy, vol. 34, p. 178, 2002.

[34] K. Kawaguchi, T. Amemiya, H. Shimizu, and Y. Hamada, "Image-guided robotic stereotactic radiotherapy for synchronous cancer of maxillary gingiva and lung," International Journal of Oral and Maxillofacial Surgery, vol. 43, pp. 692-695, 2014.

[35] M. J. Mun, D. W. Jung, C. H. Lee, and K. S. Cho, "Endoscopic removal of a huge keratocystic odontogenic tumor in maxillary sinus," Journal of Craniofacial Surgery, vol. 25, pp. 586-588, 2014.

[36] A. Weckx, J. O. Agbaje, Y. Sun, R. Jacobs, and C. Politis, "Visualization techniques of the inferior alveolar nerve (IAN): a narrative review," Surgical and Radiologic Anatomy: SRA, vol. 38, pp. 55-63, 2016.

[37] K. Ohashi, N. Sakamoto, M. Watanabe, H. Mizushima, and H. Tanaka, "Development of a telediagnosis endoscopy system over secure internet," Methods of Information in Medicine, vol. 47, pp. 157-166, 2008.

[38] S. A. Held, Y. H. Kao, and D. W. Wells, "Endoscope--an endodontic application," Journal of Endodontics, vol. 22, pp. 327-329, 1996.

[39] T. von Arx, S. Hunenbart, and D. Buser, "Endoscope- and video-assisted endodontic surgery," Quintessence international, vol. 33, pp. 255-259, 2002.

[40] Y. Yamazaki, T. Ozawa, T. Ogawa et al., "Dental fiberscope with navigation system for endodontic treatments," Studies in Health Technology and Informatics, vol. 132, pp. 562-564, 2008.

[41] M. Fujimoto, M. Okuda, S. Yoshii et al., "Endoscopic system based on intraoral camera and image processing," IEEE
Transactions on Bio-Medical Engineering, vol. 66, pp. 10261033, 2019.

[42] A. Chércoles-Ruiz, A. Sánchez-Torres, and C. E. Gay-Escoda, "Endodontic retreatment, and apical surgery versus tooth extraction and implant placement: a systematic review," Journal of Endodontics, vol. 43, pp. 679-686, 2017.

[43] R. Ordinola-Zapata and O. A. Peters, "What is of interest in Endodontology?" A bibliometric review of research published in the International Endodontic Journal and the Journal of Endodontics from, vol. 53, pp. 36-52, 2020.

[44] I. Prada, P. Micó-Muñoz, T. Giner-Lluesma, P. MicóMartínez, N. Collado-Castellano, and A. Manzano-Saiz, "Influence of microbiology on endodontic failure. Literature review," Medicina Oral, Patologia Oral Y Cirugia Bucal, vol. 24, pp. e364-e372, 2019.

[45] B. M. Cleghorn, W. H. Christie, and C. C. Dong, "Root and root canal morphology of the human permanent maxillary first molar: a literature review," Journal of Endodontics, vol. 32, pp. 813-821, 2006.

[46] H. Apotheker and G. J. Jako, "A microscope for use in dentistry," Journal of Microsurgery, vol. 3, pp. 7-10, 1981.

[47] S. M. Jang and E. Kim, "An update on endodontic microsurgery of mandibular molars," A Focused Review, vol. 57, 2021.

[48] A. Schmocker, A. Khoushabi, C. Schizas, P. E. Bourban, D. P. Pioletti, and C. Moser, "Miniature probe for the delivery and monitoring of a photopolymerizable material," Journal of Biomedical Optics, vol. 20, Article ID 127001, 2015.

[49] S. Yoshii, M. Fujimoto, M. Okuda, and C. Kitamura, "Vitro evaluation of a novel root canal endoscope for visualizing the apex of curved root canal models and an extracted tooth," Journal of Endodontics, vol. 44, pp. 1856-1861, 2018.

[50] T. Yoshioka, C. Kobayashi, and H. Suda, "Detection rate of root canal orifices with a microscope," Journal of Endodontics, vol. 28, pp. 452-453, 2002.

[51] L. A. Baldassari-Cruz, J. P. Lilly, and E. M. Rivera, "The influence of dental operating microscope in locating the mesiolingual canal orifice," Oral Surgery, Oral Medicine, Oral Pathology, Oral Radiology, and Endodontics, vol. 93, pp. 190-194, 2002.

[52] L. J. Buhrley, M. J. Barrows, E. A. BeGole, and C. S. Wenckus, "Effect of magnification on locating the MB2 canal in maxillary molars," Journal of Endodontics, vol. 28, pp. 324-327, 2002.

[53] A. Keles, C. Keskin, R. Alqawasmi, and H. Aydemir, "Accuracy of an endoscope to detect root canal anastomoses in mandibular molar teeth: a comparative study with microcomputed tomography," Acta Odontologica Scandinavica, vol. 78, pp. 433-437, 2020.

[54] L. Monaghan, S. Jadun, and J. Darcey, "Endodontic microsurgery. Part one: diagnosis, patient selection and prognoses," British Dental Journal, vol. 226, pp. 940-948, 2019.

[55] Y. Kim, D. Lee, D. V. Kim, and S. Y. Kim, "Analysis of Cause of Endodontic Failure of C-Shaped Root Canals," , Article ID 2516832, 2018.

[56] S. Bürklein and E. Schäfer, "Minimally invasive endodontics," Quintessence international, vol. 46, pp. 119-124, 2015.

[57] M. Song, H. C. Kim, W. Lee, and E. Kim, "Analysis of the cause of failure in nonsurgical endodontic treatment by microscopic inspection during endodontic microsurgery," Journal of Endodontics, vol. 37, pp. 1516-1519, 2011.

[58] A. A. Madarati, M. J. Hunter, and P. M. Dummer, "Management of intracanal separated instruments," Journal of Endodontics, vol. 39, pp. 569-581, 2013. 
[59] G. Plotino, C. H. Pameijer, N. M. Grande, and F. Somma, "Ultrasonics in endodontics: a review of the literature," Journal of Endodontics, vol. 33, pp. 81-95, 2007.

[60] T. Tsurumachi, "Current strategy for successful periradicular surgery," Journal of Oral Science, vol. 55, pp. 267-273, 2013.

[61] F. C. Setzer, S. B. Shah, M. R. Kohli, B. Karabucak, and S. Kim, "Outcome of endodontic surgery: a meta-analysis of the literature--part 1: comparison of traditional root-end surgery and endodontic microsurgery," Journal of Endodontics, vol. 36, pp. 1757-1765, 2010.

[62] T. Iwai, N. Tamai, Y. Matsui, and I. Tohnai, "Use of in-house, full-colour printed three-dimensional model for training in endoscopic periradicular surgery for molar radicular cyst," British Journal of Oral and Maxillofacial Surgery, vol. 50, pp. e41-42, 2012.

[63] B. García, M. Peñarrocha, M. A. Peñarrocha, and T. Von Arx, "Apical surgery of a maxillary molar creating a maxillary sinus window using ultrasonics: a clinical case," International Endodontic Journal, vol. 43, pp. 1054-1061, 2010.

[64] C. A. Nelson, S. G. Hossain, A. Al-Okaily, and J. Ong, "A novel vending machine for supplying root canal tools during surgery," Journal of Medical Engineering \& Technology, vol. 36, pp. 102-116, 2012.

[65] J. Dong, S. Hong, D. Ph, G. Hesselgren, and P. D. Dds, "WIP: A Study on the Development of Endodontic Micro Robot," in Proceedings of the 2006 IJME-INTERTECH Conference, Union, NJ, USA, October 2006.

[66] J. Dong and S. Y. Hong, "Design of Z Axis Actuator and quick tool change assembly for an endodontic micro robot," in Proceedings of the Asme International Mechanical Engineering Congress \& Exposition, New York, Ny, USA, June 2010.

[67] G. Hwang, A. J. Paula, E. E. Hunter et al., "Catalytic antimicrobial robots for biofilm eradication," Science robotics, vol. 4, 2019.

[68] D. F. Kinane, "Causation and pathogenesis of periodontal disease," Periodontology, vol. 25, pp. 8-20, 20002001.

[69] J. Highfield, "Diagnosis and classification of periodontal disease," Australian Dental Journal, vol. 54, no. Suppl 1, pp. S11-S26, 2009.

[70] D. A. Shanelec, "Periodontal microsurgery," Journal of esthetic and restorative dentistry: official publication of the American Academy of Esthetic Dentistry, vol. 15, pp. 402-407, 2003.

[71] S. Bittencourt, E. Del Peloso Ribeiro, E. A. Sallum, F. H. Nociti Jr., and M. Z. Casati, "Surgical microscope may enhance root coverage with subepithelial connective tissue graft: a randomized-controlled clinical trial," Journal of Periodontology, vol. 83, pp. 721-730, 2012.

[72] Y. Kuang, B. Hu, J. Chen, G. Feng, and J. Song, "Effects of periodontal endoscopy on the treatment of periodontitis: a systematic review and meta-analysis," Journal of the American Dental Association, vol. 148, pp. 750-759, 2017.

[73] C. P. Ernst, B. Willershausen, G. Driesen, P. R. Warren, and P. Hilfinger, "A robot system for evaluating plaque removal efficiency of toothbrushes in vitro," Quintessence International, vol. 28, pp. 441-445, 1997.

[74] F. Kawecki, W. P. Clafshenkel, M. Fortin, F. A. Auger, and J. Fradette, "Biomimetic tissue-engineered bone substitutes for maxillofacial and craniofacial repair," The Potential of Cell Sheet Technologies, vol. 7, Article ID e1700919, 2018.

[75] D. Kim and R. Li, "Contemporary treatment of locally advanced oral cancer," Current Treatment Options in Oncology, vol. 20, p. 32, 2019.

[76] D. B. Rodrigues and V. Castro, "Condylar hyperplasia of the temporomandibular joint: types, treatment, and surgical implications," Oral and Maxillofacial Surgery Clinics of North America, vol. 27, pp. 155-167, 2015.

[77] U. Prechel, P. Ottl, O. M. Ahlers, and A. Neff, "The treatment of temporomandibular joint dislocation," Deutsches Arzteblatt international, vol. 115, pp. 59-64, 2018.

[78] C. Porcheri and T. A. Mitsiadis, "Physiology, pathology and regeneration of salivary glands," Cells, vol. 8, 2019.

[79] Y. Ahn, "Devices for minimally-invasive microdiscectomy: current status and future prospects," Expert Review of Medical Devices, vol. 17, pp. 131-138, 2020.

[80] Z. Wang, K. Sano, T. Inokuchi et al., "The free deltoid flap: microscopic anatomy studies and clinical application to oral cavity reconstruction," Plastic and Reconstructive Surgery, vol. 112, pp. 404-411, 2003.

[81] M. H. Seo, S. M. Kim, F. Huan, H. Myoung, J. H. Lee, and S. K. Lee, "Analysis of microvascular free flap failure focusing on the microscopic findings of the anastomosed vessels," Journal of Craniofacial Surgery, vol. 26, pp. 2047-2051, 2015.

[82] D. R. Ashworth, N. M. Whear, and V. Fan, "Radial free flaps using loupe magnification: audit of 97 cases of orofacial reconstruction," British Journal of Oral and Maxillofacial Surgery, vol. 42, pp. 36-37, 2004.

[83] T. W. Rutner, V. B. Ziccardi, and M. N. Janal, "Long-term outcome assessment for lingual nerve microsurgery," Journal of Oral and Maxillofacial Surgery: Official Journal of the American Association of Oral and Maxillofacial Surgeons, vol. 63, pp. 1145-1149, 2005.

[84] B. Xie, S. Zhang, and Y. Liu, "Endoscopic-assisted repair of spontaneous temporomandibular joint herniation through a transcanal approach," Otology \& Neurotology: official publication of the American Otological Society, American Neurotology Society [and] European Academy of Otology and Neurotology, vol. 40, pp. 772-776, 2019.

[85] R. Elledge, B. Speculand, J. Green, and A. Attard, “Training in surgery of the temporomandibular joint: perceptions of trainees in oral and maxillofacial surgery in the United Kingdom," British Journal of Oral and Maxillofacial Surgery, vol. 54, pp. 941-945, 2016.

[86] Y. Wu, F. Wang, S. Fan, and J. K. Chow, "Robotics in dental implantology," Oral and Maxillofacial Surgery Clinics of North America, vol. 31, pp. 513-518, 2019.

[87] A. C. Nichols, J. Theurer, E. Prisman et al., "Radiotherapy versus transoral robotic surgery and neck dissection for oropharyngeal squamous cell carcinoma (ORATOR): an open-label, phase 2, randomised trial," The Lancet Oncology, vol. 20, pp. 1349-1359, 2019.

[88] A. W. Barrett, M. Villarroel Dorrego, T. A. Hodgson et al., "The histopathology of syphilis of the oral mucosa," Journal of Oral Pathology \& Medicine: Official Publication of the International Association of Oral Pathologists and the American Academy of Oral Pathology, vol. 33, pp. 286-291, 2004.

[89] C. W. van Wyk, H. A. Seedat, and V. M. Phillips, "Collagen in submucous fibrosis: an electron-microscopic study," Journal of Oral Pathology \& Medicine: Official Publication of the International Association of Oral Pathologists and the American Academy of Oral Pathology, vol. 19, pp. 182-187, 1990.

[90] S. Hallikerimath, G. Sapra, A. Kale, and P. R. Malur, "Cytomorphometric analysis and assessment of periodic acid Schiff positivity of exfoliated cells from apparently normal buccal mucosa of type 2 diabetic patients," Acta Cytologica, vol. 55, pp. 197-202, 2011.

[91] F. Saeed, N. Muhammad, A. S. Khan et al., "Prosthodontics dental materials: from conventional to unconventional," 
Materials science \& engineering. C, Materials for biological applications, vol. 106, Article ID 110167, 2020.

[92] J. Chen, H. Cai, L. Suo, Y. Xue, J. Wang, and Q. Wan, “A systematic review of the survival and complication rates of inlay-retained fixed dental prostheses," Journal of Dentistry, vol. 59, pp. 2-10, 2017.

[93] C. A. Barwacz, M. Hernandez, and R. H. Husemann, "Minimally invasive preparation and design of a cantilevered, allceramic, resin-bonded, fixed partial denture in the esthetic zone: a case report and descriptive review," Journal of esthetic and restorative dentistry: official publication of the American Academy of Esthetic Dentistry, vol. 26, pp. 314-323, 2014.

[94] T. C. T. van Riet, K. T. H. Chin Jen Sem, J. T. F. Ho, R. Spijker, J. Kober, and J. de Lange, "Robot technology in dentistry, part two of a systematic review: an overview of initiatives," Dental Materials: Official Publication of the Academy of Dental Materials, vol. 37, pp. 1227-1236, 2021.

[95] F. Yuan, Y. Wang, Y. Zhang, Y. Sun, D. Wang, and P. Lyu, "An automatic tooth preparation technique: a preliminary study," Scientific Reports, vol. 6, Article ID 25281, 2016.

[96] R. Boesecke, J. Brief, J. Raczkowsky et al., Robot Assistant for Dental Implantology, Springer, Berlin, Germany, pp. 1302-1303, 2001.

[97] X. Sun, Y. Yoon, J. Li, and F. D. McKenzie, "Automated image-guided surgery for common and complex dental implants," Journal of Medical Engineering \& Technology, vol. 38, pp. 251-259, 2014.

[98] K. Yu, S. Uozumi, K. Ohnishi, S. Usuda, H. Kawana, and T. Nakagawa, "Stereo vision based robot navigation system using modulated potential field for implant surgery," in Proceedings of the 2015 IEEE International Conference on Industrial Technology (ICIT), pp. 493-498, Seville, Spain, March 2015.

[99] P. S. Mozer, "Accuracy and deviation analysis of static and robotic guided implant surgery: a case study," The International Journal of Oral \& Maxillofacial Implants, vol. 35, pp. e86-e90, 2020.

[100] G. B. Carr, "Microscopic photography for the restorative dentist," Journal of esthetic and restorative dentistry: official publication of the American Academy of Esthetic Dentistry, vol. 15, pp. 417-425, 2003.

[101] G. C. Burdea, S. M. Dunn, and G. Levy, "Evaluation of robotbased registration for subtraction radiography," Medical Image Analysis, vol. 3, pp. 265-274, 1999.

[102] S. Kim and S. Baek, "The microscope and endodontics," Dental Clinics of North America, vol. 48, pp. 11-18, 2004.

[103] M. J. Hayes, J. A. Taylor, and D. R. Smith, "Predictors of work-related musculoskeletal disorders among dental hygienists," International Journal of Dental hygiene, vol. 10, pp. 265-269, 2012.

[104] M. Bud, R. Pricope, and R. C. Pop, "Comparative analysis of preclinical dental students' working postures using dental loupes and dental operating microscope," vol. 25, pp. 516-523, 2021.

[105] K. Sweeney and M. Mackey, "The effectiveness of ergonomics interventions in reducing upper limb work-related musculoskeletal pain and dysfunction in sonographers, surgeons and dentists: a systematic review," vol. 64, pp. 1-38, 2021. 